\title{
VALUES OF $L$-FUNCTIONS AT THE CRITICAL POINT
}

\author{
LIEM MAI
}

(Communicated by Dennis A. Hejhal)

ABSTRACT. For a discriminant $D$ of a binary quadratic form, we study the average value of $L\left(s, \varepsilon_{D}\right)$ at the critical point $\frac{1}{2}$ where $\varepsilon_{D}$ is defined by $\mathrm{W}$. Kohnen and D. Zagier :

$$
\varepsilon_{D}(n)=\sum_{\substack{g>0 \\ g\left|\delta, g^{2}\right| n \\\left(\delta / 8, n / g^{2}\right)=1}}\left(\frac{D_{0}}{g^{-2} n}\right) g
$$

for $n \in \mathbb{N}$ and $D=D_{0} \delta^{2}, D_{0}$ a fundamental discriminant and $\delta \in \mathbb{N}$. When $D=D_{0}, L\left(s, \varepsilon_{D_{0}}\right)$ is the Dirichlet series $L\left(s,\left(\frac{D_{0}}{s}\right)\right)$. We derive an asymptotic formula for $\sum_{D} L\left(\frac{1}{2}, \varepsilon_{D}\right)$, where the sum runs over all discriminants $D \in$ $(0, Y]$ or $[-Y, 0)$.

Let $D$ be any discriminant of a binary quadratic form, i.e., $D \in \mathbb{Z}$ and $D \equiv 0,1(\bmod 4)$. The full modular group $\Gamma=\mathrm{SL}_{2}(\mathbb{Z}) / \pm I$ operates on the set of binary quadratic forms $f(u, v)$ by

$$
(\gamma \circ f)(u, v)=f(a u+c v, b u+d v)
$$

for $\gamma=\left(\begin{array}{ll}a & b \\ c & d\end{array}\right)$.

In [10], Zagier defined

$$
\zeta(s, D)=\sum_{f} \sum_{\substack{(m, n) \in \mathbb{Z}^{2} / \operatorname{Ant}(\Omega) \\(m, n)>0}} \frac{1}{f(m, n)^{s}}
$$

where the first sum runs over all $\Gamma$ equivalence classes of binary quadratic forms $f(u, v)$ of discriminant $D$ and the second over all inequivalent pairs of integers with respect to the group of units $\operatorname{Aut}(f)=\{\gamma \in \Gamma: \gamma f=f\}$. He proved that

$$
\zeta(s, D)=\zeta(2 s) \sum_{n=1}^{\infty} a_{n} n^{-s}
$$

Received by the editors February 3, 1993.

1991 Mathematics Subject Classification. Primary 11C54.

$K e y$ words and phrases. $L$-functions, discriminants. 
where $a_{n}$ is the number of solutions $x(\bmod 2 n)$ of the congruence $x^{2} \equiv D$ $(\bmod 4 n)$ and $\zeta(s, D)$ can be expressed in terms of standard Dirichlet series:

$$
\begin{aligned}
\zeta(s, D) & =\zeta(s) L_{D}(s) \\
& =\zeta(s) L_{D_{0}}(s) \sum_{d \mid \delta} \mu(d)\left(\frac{D_{0}}{d}\right) d^{-s} \sigma_{1-2 s}\left(\frac{\delta}{d}\right)
\end{aligned}
$$

where $D=D_{0} \delta^{2}, D_{0}$ is a fundamental discriminant, $\left(\frac{D_{0}}{-}\right)$ is the Kronecker symbol, $L_{D_{0}}(s)$ is the Dirichlet series attached to $\left(\frac{D_{0}}{2}\right)$, and $\sigma_{k}(m)=\sum_{d \mid m} d^{k}$.

In [5], Kohnen and Zagier defined, for $D=D_{0} \delta^{2}$ :

$$
\varepsilon_{D}(n)=\sum_{\substack{g>0 \\ g\left|\delta, 8^{2}\right| n \\\left(\delta / 8, n / g^{2}\right)=1}}\left(\frac{D_{0}}{g^{-2} n}\right) g
$$

then

$$
L_{D}(s)=\sum_{n=1}^{\infty} \varepsilon_{D}(n) n^{-s}=L\left(s, \varepsilon_{D}\right) \quad(\text { say }) .
$$

Note that $\varepsilon_{D}$ is slightly different from $\psi_{D}()=.\left(\frac{D}{.}\right)$. If $D=D_{0}$, a fundamental discriminant, then $\varepsilon_{D}(n)=\psi_{D_{0}}(n)=\left(\frac{D_{0}}{n}\right)$ and $L\left(s, \varepsilon_{D}\right)$ turns out to be the Dirichlet series $L_{D_{0}}(s)=L\left(s, \psi_{D_{0}}\right)=L\left(s,\left(\frac{D_{0}}{.}\right)\right)$; and if $D=D_{0} \delta^{2}$ where $D_{0}$ is a fundamental discriminant, it equals the product of $L_{D_{0}}(s)$ with a finite Euler product over the divisors of $\delta$.

For $\psi_{D}$, using the theory of quadratic forms, Siegel (see [8]) proved that

$$
\sum_{0<D \leq Y} L\left(1, \psi_{D}\right)=C Y+O\left(Y^{1 / 2} \log Y\right)
$$

and Vinogradov and Tahtadzjan (see [9]), by using exponential sums, proved that

$$
\sum_{0<D \leq Y} L\left(\frac{1}{2}, \psi_{D}\right)=Y\left\{C \log Y+C^{\prime}\right\}+O\left(Y^{1 / 2+\varepsilon}\right)
$$

where $C$ and $C^{\prime}$ are explicit constants.

For fundamental discriminants $D_{0}$, using a mean square estimate for quadratic character sums, Jutila (see [4]) proved a conjecture by Goldfeld and Viola (see [3]) which states that

$$
\sum_{\substack{0< \pm D_{0} \leq Y \\ D_{0} \text { fund. disc. }}} L_{D_{0}}\left(\frac{1}{2}\right)=Y\left\{C_{1} \log Y+C_{1}^{\prime}\right\}+O\left(Y^{3 / 4+\varepsilon}\right)
$$

and later, using Eisenstein series of half integral weight, Goldfeld and Hoffstein (see [2]) improved the error term:

$$
\sum_{\substack{0< \pm D_{0} \leq Y \\ D_{0} \text { fund dise }}} L_{D_{0}}\left(\frac{1}{2}\right)=Y\left\{C_{1} \log Y+C_{1}^{\prime}\right\}+O\left(Y^{19 / 32+\varepsilon}\right)
$$

where $C, C^{\prime}$ are explicit constants. 
In this paper, we will prove a similar result for $\varepsilon_{D}$, where $D$ is a discriminant, using the nice functional equation satisfied by $L\left(s, \varepsilon_{D}\right)$ :

$$
L\left(\frac{1}{2}+s, \varepsilon_{D}\right)=D^{s} \pi^{s} \frac{\Gamma\left(\frac{1}{4}-\frac{s}{2}\right)}{\Gamma\left(\frac{1}{4}+\frac{s}{2}\right)} L\left(\frac{1}{2}-s, \varepsilon_{D}\right)
$$

as they are Fourier coefficients of Eisenstein series (see [10], for example). The method is far simpler than the previous ones, and the error term is better than the one obtained by Jutila.

Theorem 1.

$$
\sum_{\substack{0<D<Y \\ D \equiv 0,1(\bmod 4) \\ i \\ 2^{2} \mid D}} L\left(\frac{1}{2}, \varepsilon_{D}\right)=C_{1}(t) Y \log Y+C_{2}(t) Y+O\left(Y^{2 / 3} \log Y\right),
$$

where

and

$$
C_{1}(t)= \begin{cases}\frac{5}{16 t^{2}} \sum_{t_{1} \mid t} \frac{\phi\left(t_{1}\right)}{t_{1}} & \text { if } t \text { is odd }, \\ \frac{1}{2 t^{2}} \sum_{t_{1} \mid t} \frac{\phi\left(t_{1}\right)}{t_{1}} & \text { if } t \text { is even }\end{cases}
$$

$$
C_{2}(t)=\left\{\begin{array}{c}
\frac{5}{16 t^{2}} \sum_{t_{1} \mid t} \frac{\phi\left(t_{1}\right)}{t_{1}}\left(4 \gamma-\log \pi-1-\frac{2 \log 4}{5}\right. \\
\left.+\frac{\Gamma^{\prime}}{\Gamma}\left(\frac{1}{4}\right)-4 \log \left(\frac{t}{t_{1}}\right)+4 \sum_{p \mid t_{1}} \frac{\log p}{p-1}\right) \quad \text { if } t \text { is odd }, \\
\frac{1}{2 t^{2}} \sum_{t_{1} \mid t} \frac{\phi\left(t_{1}\right)}{t_{1}}(+4 \gamma-\log \pi-1 \\
\left.+\frac{\Gamma^{\prime}}{\Gamma}\left(\frac{1}{4}\right)-4 \log \left(\frac{t}{t_{1}}\right)+4 \sum_{p \mid t_{1}} \frac{\log p}{p-1}\right) \quad \text { if } t \text { is even. }
\end{array}\right.
$$

Proof. We begin with the integral

$$
\int_{(\sigma)} L\left(\frac{1}{2}+s, \varepsilon_{D}\right) X^{s} \Gamma(s) d s=\sum_{n=1}^{\infty} \varepsilon_{D}(n) \frac{1}{\sqrt{n}} e^{-n / X}
$$

where $\Re(s)=\sigma>1$.

Moving the line of integration to the left to a line $\Re(s)=-\eta$, we get

$$
L\left(\frac{1}{2}, \varepsilon_{D}\right)+\frac{1}{2 \pi i} \int_{(-\eta)} L\left(\frac{1}{2}+s, \varepsilon_{D}\right) X^{s} \Gamma(s) d s .
$$

Summing over all discriminants $D, 0<D \leq Y, t^{2} \mid D$, we have

$$
\begin{aligned}
& \sum_{\substack{D<D \leq Y \\
D \equiv 0,1 \operatorname{mod4} \\
t^{2} \mid D}} L\left(\frac{1}{2}, \varepsilon_{D}\right)=\sum_{\substack{0 \leq D \leq Y \\
D \equiv 0,1(\bmod ) \\
i^{2} \mid D}} \sum_{n=1}^{\infty} \varepsilon_{D}(n) \frac{1}{\sqrt{n}} e^{-n / X} \\
& -\frac{1}{2 \pi i} \int_{(-\eta)} \sum_{\substack{0<D \leq Y \\
D \equiv 0,1 \text { (mod4) } \\
i^{2} \mid D}} L\left(\frac{1}{2}+s, \varepsilon_{D}\right) X^{s} \Gamma(s) d s \\
& =S+I \text {. }
\end{aligned}
$$

At first, we assume that $(t, 2)=1$. 
For the sum $S$, we write $S=S_{\text {main }}+S_{\text {error }}$, where the first sum is over all squares and the latter over all nonsquares.

For the sum $S_{\text {main }}$, we have

$$
\begin{aligned}
& S_{\text {main }}=\sum_{m=1}^{\infty} \frac{1}{m} e^{-m^{2} / X} \sum_{\substack{0<D \leq Y \\
D \equiv 0,1(\bmod 4) \\
t^{2} \mid D}} \varepsilon_{D}\left(m^{2}\right)
\end{aligned}
$$

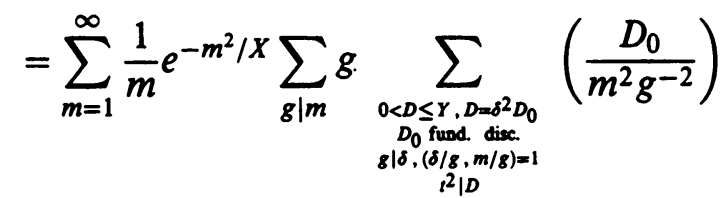

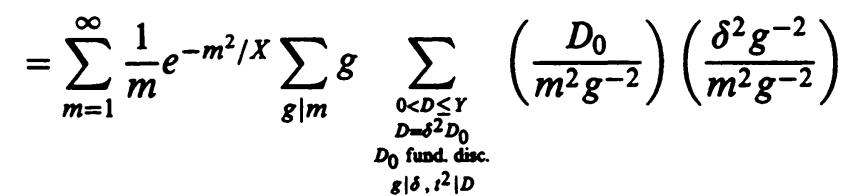

$$
\begin{aligned}
& =\sum_{m=1}^{\infty} \frac{1}{m} e^{-m^{2} / X} \sum_{g \mid m} g\left(\sum_{\substack{0<D_{1}<Y / g^{2} \\
g_{1}^{2} D_{1}=0,1(4) \\
g^{2} D_{1}=0\left(r^{2}\right)}}\left(\frac{D_{1}}{m^{2} g^{-2}}\right)\right) \text {. }
\end{aligned}
$$

For each $g \mid m$, let $(g, t)=t_{1}$; then $g=g_{1} t_{1},\left(g_{1}, t\right)=1$, and $t=t_{1} t_{2}$. We have

$$
S_{\text {main }}=\sum_{m=1}^{\infty} \frac{1}{m} e^{-m^{2} / X} \sum_{\substack{g \mid m \\\left(m / g, t_{2}\right)=1}} g \sum_{\substack{0<D_{1}<Y / g^{2} \\ g^{2} D_{1}=0,1(4) \\ g_{1}^{2} D_{1}=\alpha\left(\frac{2}{2}\right)}}\left(\frac{D_{1}}{m^{2} g^{-2}}\right) .
$$

If $g$ is even, the innermost sum is

$$
\sum_{\substack{0<D_{1}<r / g^{2} \\ g_{1}^{2} D_{1}=O\left(c_{2}^{2}\right) \\\left(D_{1}, m / g\right)=1}} 1=\frac{\phi\left(m g^{-1}\right)}{m g^{-1}} \frac{Y}{g^{2} t_{2}^{2}}+O\left(\mathrm{~d}\left(m g^{-1}\right)\right),
$$

and if $g$ is odd, it is

$$
\sum_{\substack{0<D_{1}<Y / g^{2} \\ D_{1}=0.1(4) \\ g_{1}^{2} D_{1}=\alpha\left(t_{2}^{2}\right) \\\left(D_{1}, m / g\right)=1}} 1=\frac{1}{2} \frac{\phi\left(m g^{-1}\right)}{m g^{-1}} \frac{Y}{g^{2} t_{2}^{2}}+O\left(d\left(m g^{-1}\right)\right)
$$

where $\mathrm{d}(n)=$ \# of divisors of $n$. Recall that $g$ is odd if and only if $g_{1}$ is 
odd, as $g=g_{1} t_{1}$ and $t_{1}$ is odd. Therefore,

$$
\begin{aligned}
& S_{\text {main }}=\sum_{m=1}^{\infty} \frac{1}{m} e^{-m^{2} / X}\left\{\sum_{\substack{g \mid m \\
\left(\frac{m}{g}, t_{2}\right)=1 \\
g \text { is even }}} \frac{\phi\left(m g^{-1}\right)}{m g^{-1}} \frac{Y}{g t_{2}^{2}}+\sum_{\substack{g \mid m \\
\left(\frac{m}{g}, t_{2}\right)=1 \\
g \text { is odd }}} \frac{\phi\left(m g^{-1}\right)}{m g^{-1}} \frac{1}{2} \frac{Y}{g t_{2}^{2}}\right\} \\
& +\sum_{m=1}^{\infty} \frac{1}{m} e^{-m^{2} / X}\left(O\left(\sum_{g \mid m} g \mathrm{~d}\left(m g^{-1}\right)\right)\right) \text {. }
\end{aligned}
$$

Note that the condition $\left(m / g, t_{2}\right)=\left(m / g_{1} t_{1}, t_{2}\right)=1$ implies that $m=$ $m_{1} t_{1}$, where $\left(m_{1}, t_{2}\right)=1$. Therefore, we can write the sum $S_{\text {main }}$ as

$$
\begin{aligned}
& \sum_{t_{1} \mid t} \sum_{\substack{m_{1}=1 \\
\left(m_{1}, t_{2}\right)=1}}^{\infty} \frac{1}{m_{1} t_{1}} e^{-m_{1}^{2} t_{1}^{2} / X} \\
& \times\left\{\sum_{\substack{g_{1} \mid m_{1} \\
\left(\frac{m_{1}}{g_{1}}, t_{2}\right)=1 \\
g_{1} \text { is even }}} \frac{\phi\left(m_{1} g_{1}^{-1}\right)}{m_{1} g_{1}^{-1}} \frac{Y}{g_{1} t_{1} t_{2}^{2}}+\sum_{\substack{g_{1} \mid m_{1} \\
\left(\frac{m_{1}}{s_{1}}, t_{2}\right)=1 \\
g_{1} \text { is odd }}} \frac{\phi\left(m_{1} g_{1}^{-1}\right)}{m_{1} g_{1}^{-1}} \frac{1}{2} \frac{Y}{g_{1} t_{1} t_{2}^{2}}\right\} \\
& +\sum_{t_{1} \mid t} \sum_{\substack{m_{1}=1 \\
\left(m_{1}, t_{2}\right)=1}}^{\infty} \frac{1}{m_{1} t_{1}} e^{-m_{1}^{2} t_{1}^{2} / X} O\left(\sum_{g_{1} \mid m_{1}} g_{1} t_{1} \mathbf{d}\left(\frac{m_{1}}{g_{1}}\right)\right) \\
& =\sum_{t_{1} \mid t}\left\{\sum_{\substack{g_{1} \\
\left(g_{1}, t_{2}\right)=1 \\
g_{1} \text { is even }}} \sum_{\substack{\left.h_{1}=1 \\
h_{1}, t_{2}\right)=1}}^{\infty} \frac{1}{g_{1}^{2}} \frac{\phi\left(h_{1}\right)}{h_{1}^{2}} \frac{Y}{t^{2}} e^{-g_{1}^{2} h_{1}^{2} t_{1}^{2} / X}\right. \\
& \left.+\sum_{\substack{g_{1} \\
\left(g_{1}, t_{2}\right)=1 \\
g_{1} \text { is odd }}} \sum_{\substack{h_{1}=1 \\
\left(h_{1}, t_{2}\right)=1}}^{\infty} \frac{1}{2} \frac{1}{g_{1}^{2}} \frac{\phi\left(h_{1}\right)}{h_{1}^{2}} \frac{Y}{t^{2}} e^{-g_{1}^{2} h_{1}^{2} t_{1}^{2} / X}\right\} \\
& +O\left(\sum_{t_{1} \mid t} \sum_{g_{1}} \sum_{h_{1}} \frac{1}{h_{1}} \mathrm{~d}\left(h_{1}\right) e^{-g_{1}^{2} h_{1}^{2} t_{1}^{2} / X}\right) .
\end{aligned}
$$

The last term is $O\left(\mathrm{~d}(t) X^{1 / 2}(\log X)^{2}\right)$.

The first sum is

$$
\begin{aligned}
& \frac{1}{4} \frac{Y}{t^{2}} \sum_{t_{1} \mid t} \sum_{\substack{s_{2}=1 \\
\left(g_{2}, t_{2}\right)=1}}^{\infty} \frac{1}{g_{2}^{2}} \sum_{\substack{h_{1}=1 \\
\left(h_{1}, t_{2}\right)=1}}^{\infty} \frac{\phi\left(h_{1}\right)}{h_{1}^{2}} e^{-g_{2}^{2} h_{1}^{2} /\left(X / 4 t_{1}^{2}\right)} \\
& \quad=\frac{1}{4} \frac{Y}{t^{2}} \sum_{t_{1} \mid t} \int_{(\sigma)} \zeta(2 s+1) \prod_{p \mid t_{2}}\left(1-\frac{1}{p^{2 s+1}}\right)\left(\frac{X}{4 t_{1}^{2}}\right)^{s} \Gamma(s) d s .
\end{aligned}
$$


We move the line of integration to the left to the line $\Re(s)=-1+\frac{1}{\log X}$. Using the Laurent expansions at $s=0$, we have:

$$
\begin{aligned}
\zeta(1+2 s) & =\frac{1}{2 s}+\gamma+\cdots, \\
\left(\frac{X}{4 t_{1}^{2}}\right)^{s} & =1+\log \left(\frac{X}{4 t_{1}^{2}}\right) s+\cdots, \\
\Gamma(s) & =\frac{1}{s}-\gamma+\cdots, \\
\prod_{p \mid t_{2}}\left(1-\frac{1}{p^{2 s+1}}\right) & =\frac{\phi\left(t_{2}\right)}{t_{2}}+\frac{\phi\left(t_{2}\right)}{t_{2}}\left(\sum_{p \mid t_{2}} \frac{2 \log p}{p-1}\right) s+\cdots
\end{aligned}
$$

where $\gamma$ is the Euler constant. Then the first sum is

$$
\begin{aligned}
& \frac{1}{4} \frac{Y}{t^{2}} \sum_{t_{1} \mid t} \frac{\phi\left(t_{2}\right)}{t_{2}}\left(\frac{1}{2}(\log X)+\left(\frac{\gamma}{2}-\frac{1}{2} \log \left(4 t_{1}^{2}\right)+\sum_{p \mid t_{2}} \frac{\log p}{p-1}\right)\right) \\
& +O\left(\frac{Y}{X} \sum_{t_{1} \mid t} \frac{1}{t_{1}}\right)+O\left(\mathbf{d}(t) X^{1 / 2}(\log X)^{2}\right) \\
& =\frac{1}{4} \frac{Y}{t^{2}} \sum_{t_{1} \mid t} \frac{\phi\left(t_{1}\right)}{t_{1}}\left(\frac{1}{2}(\log X)+\left(\frac{\gamma}{2}-\frac{1}{2} \log \left(4 t_{2}^{2}\right)+\sum_{p \mid t_{1}} \frac{\log p}{p-1}\right)\right) \\
& +O\left(\frac{Y}{X} \sum_{t_{1} \mid t} \frac{1}{t_{1}}\right)+O\left(\mathbf{d}(t) X^{1 / 2}(\log X)^{2}\right)
\end{aligned}
$$

as $t=t_{1} t_{2}, t_{1}$ and $t_{2}$ are interchangeable.

The second term is

$$
\begin{aligned}
& \frac{1}{2} \sum_{t_{1} \mid t}\left(\sum_{\substack{g_{1}=1 \\
\left(s_{1}, t_{2}\right)=1}}^{\infty} \sum_{h_{1}}-\sum_{\substack{\left.g_{1}=1 \\
g_{1}, l_{2}\right)=1 \\
g_{1} \text { is even }}}^{\infty} \sum_{h_{1}}\right) \\
& =\frac{Y}{t^{2}} \sum_{t_{1} \mid t} \frac{\phi\left(t_{1}\right)}{t_{1}}\left(\frac{3}{16}(\log X)+\frac{3}{16} \gamma+\frac{\log 4}{16}-\frac{3}{8}\left(\log t_{2}\right)+\frac{3}{8} \sum_{p \mid t_{1}} \frac{\log p}{p-1}\right) \\
& +O\left(\frac{Y}{X} \sum_{t_{1} \mid t} \frac{1}{t_{1}}\right)+O\left(\mathrm{~d}(t) X^{1 / 2}(\log X)^{2}\right) .
\end{aligned}
$$

Therefore,

$$
\begin{aligned}
S_{\text {main }}=\frac{Y}{t^{2}} \sum_{t_{1} \mid t} \frac{\phi\left(t_{1}\right)}{t_{1}}\left(\frac{5}{16}(\log X)+\frac{5}{16} \gamma-\frac{\log 4}{16}-\frac{5}{8}\left(\log t_{2}\right)+\frac{5}{8} \sum_{p \mid t_{1}} \frac{\log p}{p-1}\right) \\
+O\left(\frac{Y}{X} \sum_{t_{1} \mid t} \frac{1}{t_{1}}\right)+O\left(\mathrm{~d}(t) X^{1 / 2}(\log X)^{2}\right) .
\end{aligned}
$$


Next, for the sum $S_{\text {error }}$, we have

$$
S_{\text {error }}=\sum_{\substack{n=1 \\ n \text { is nonsquare }}}^{\infty} \frac{1}{\sqrt{n}} e^{-n / X}\left(\sum_{\substack{0<D \leq Y \\ D \equiv 0,1(\bmod 4) \\ i^{2} \mid D}} \varepsilon_{D}(n)\right) .
$$

We need a lemma:

Lemma. For a nonsquare integer $n$, we have

$$
\sum_{\substack{0<D \leq \gamma \\ D \equiv 0,1(\bmod 4) \\ t^{2} \mid D}} \varepsilon_{D}(n)=O(\sqrt{n}(\log n) \gamma(n))
$$

where $\gamma(n)=\sum_{g^{2} \mid n} 1$.

Proof.

$$
\begin{aligned}
& \sum_{\substack{0<D \leq Y \\
D \equiv 0,1(\bmod 4) \\
i^{2} \mid D}} \varepsilon_{D}(n)=\sum_{\substack{0<D \leq Y \\
D \equiv 0,1(\bmod 4) \\
t^{2} \mid D}} \sum_{\substack{g \\
g^{2}|n, g| \delta \\
\text { for } D=\delta^{2} D_{0} \\
\left(\delta / g, n / g^{2}\right)=1}}\left(\frac{D_{0}}{n g^{-2}}\right) g \\
& =\sum_{g^{2} \mid n} g\left(\sum_{\substack{0<D \leq Y \\
g^{2}\left|D \\
D=0,1(4) \\
t^{2}\right| D}}\left(\frac{D_{0}}{n g^{-2}}\right)\left(\frac{\delta^{2} g^{-2}}{n g^{-2}}\right)\right) \\
& =\sum_{g^{2} \mid n} g\left(\sum_{\substack{0<D_{1} \leq Y / g^{2} \\
g^{2} D_{1}=0,1(\bmod 4) \\
g^{2} D_{1}=0\left(2^{2}\right)}}\left(\frac{D_{1}}{n g^{-2}}\right)\right)=O\left(\sum_{g^{2} \mid n} g \frac{\sqrt{n}}{g} \log n\right)
\end{aligned}
$$

by the Polya-Vinogradov inequality.

Hence,

$$
\sum_{\substack{0<D \leq Y \\ D \equiv 0,1(\bmod 4) \\ t^{2} \mid D}} \varepsilon_{D}(n)=O(\sqrt{n}(\log n) \gamma(n)) .
$$

Returning to our sum $S_{\text {error }}$, we have

$$
\begin{aligned}
S_{\text {error }} & =O\left(\sum_{\substack{n=1 \\
n \text { is soosquare }}}^{\infty} \frac{1}{\sqrt{n}} e^{-n / X} \sqrt{n}(\log n) \gamma(n)\right) \\
& =O\left(\sum_{n \leq X}(\log n) \gamma(n)\right)=O\left(\log X \sum_{n \leq X} \sum_{g^{2} \mid n} 1\right) \\
& =O\left(\log X \sum_{g \leq \sqrt{X}} \sum_{h \leq X / g^{2}} 1\right)=O(X \log X) .
\end{aligned}
$$


Now for the integral $I$, we have

$$
I=-\frac{1}{2 \pi i} \int_{(-\eta)} \sum_{\substack{0<D \leq Y \\ D \equiv 0,1(\bmod 4) \\ i^{2} \mid D}} L\left(\frac{1}{2}+s, \varepsilon_{D}\right) X^{s} \Gamma(s) d s
$$

where $L\left(s, \varepsilon_{D}\right)$ satisfies a functional equation

$$
L\left(\frac{1}{2}+s, \varepsilon_{D}\right)=D^{s} \pi^{s} \frac{\Gamma\left(\frac{1}{4}-\frac{s}{2}\right)}{\Gamma\left(\frac{1}{4}+\frac{s}{2}\right)} L\left(\frac{1}{2}-s, \varepsilon_{D}\right) .
$$

Substituting into our integral $I$, we have

$$
\begin{aligned}
I & =-\frac{1}{2 \pi i} \int_{(-\eta)} \sum_{\substack{0<D \leq Y \\
D \equiv 0,1 \text { (mod4) } \\
i^{2} \mid D}} L\left(\frac{1}{2}-s, \varepsilon_{D}\right) D^{-s} \pi^{s} \frac{\Gamma\left(\frac{1}{4}-\frac{s}{2}\right)}{\Gamma\left(\frac{1}{4}+\frac{s}{2}\right)} X^{s} \Gamma(s) d s \\
& =-\frac{1}{2 \pi i} \int_{(-\eta)} \sum_{\substack{0<D \leq Y \\
D \equiv 0,1(\bmod ) \\
i^{2} \mid D}} \sum_{n=1}^{\infty} \frac{\varepsilon_{D}(n)}{n^{1 / 2-s}} D^{-s} \pi^{s} X^{s} \frac{\Gamma\left(\frac{1}{4}-\frac{s}{2}\right)}{\Gamma\left(\frac{1}{4}+\frac{s}{2}\right)} \Gamma(s) d s \\
& =I_{\text {main }}+I_{\text {error }}
\end{aligned}
$$

For the integral $I_{\text {main }}$ which corresponds to squares $n=m^{2}$, we have

$$
\sum_{\substack{\alpha<D \leq Y \\ D \equiv 0,1(\operatorname{mos} d) \\ i^{2} \mid D}} \varepsilon_{D}\left(m^{2}\right) D^{-s}=\sum_{g \mid m} g^{1-2 s}\left(\sum_{\substack{0<D_{1}<Y / g^{2} \\ g^{2} D_{1}=0,1(\bmod ) \\ g^{2} D_{1}=0\left(2^{2}\right)}} \chi_{0}^{\left(m g^{-1}\right)}\left(D_{1}\right) D_{1}^{-s}\right)
$$

where $\chi_{0}^{\left(m g^{-1}\right)}(\cdot)$ is the principal character modulo $m g^{-1}$.

For each $g$, let $(g, t)=t_{1}$; then $g=g_{1} t_{1}, t=t_{1} t_{2}$, and the above sum is

$$
\begin{aligned}
& =\sum_{t_{1} \mid t} \sum_{\substack{g \mid m \\
\left(m / g, t_{2}\right)=1}} g^{1-2 s}\left(\sum_{\substack{0<D_{1}<Y / g^{2} \\
g^{2} D_{1}=0,1(\operatorname{mos}) \\
g^{2} D_{1}=0\left(t^{2}\right)}} \chi_{0}^{\left(m g^{-1}\right)}\left(D_{1}\right) D_{1}^{-s}\right)
\end{aligned}
$$

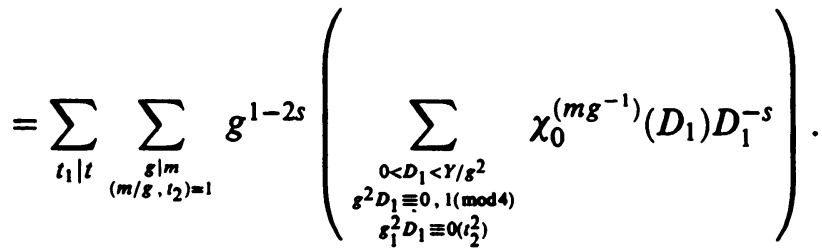


VALUES OF $L$-FUNCTIONS AT THE CRITICAL POINT

423

The innermost sum for $g$ even, by partial summation, is

$$
\frac{\phi\left(m g^{-1}\right)}{m g^{-1}}\left(\frac{Y}{g^{2} t_{2}^{2}}\right)^{1-s} \frac{t_{2}^{-2 s}}{1-s}+O\left(Y^{\eta} g^{-2 \eta} \mathbf{d}\left(\frac{m}{g}\right)(|s|+1)\right)
$$

on $\Re(s)=-\eta$. For $g$ is odd, it is

$$
\frac{1}{2} \frac{\phi\left(m g^{-1}\right.}{m g^{-1}}\left(\frac{Y}{g^{2} t_{2}^{2}}\right)^{1-s} \frac{t_{2}^{-2 s}}{1-s}+O\left(Y^{\eta} g^{-2 \eta} \mathbf{d}\left(\frac{m}{g}\right)(|s|+1)\right) .
$$

Then

$$
\begin{aligned}
& \sum_{\substack{0<D \leq Y \\
D \equiv 0,1 \text { (mod) } \\
i^{2} \mid D}} \sum_{m=1}^{\infty} \frac{\varepsilon_{D}\left(m^{2}\right)}{m^{1-2 s}} D^{-s} \\
& =\sum_{t_{1} \mid t} \sum_{\substack{m_{1}=1 \\
\left(m_{1}, t_{2}\right)=1}}^{\infty}\left\{\sum_{\substack{g_{1} \mid m_{1} \\
\left(\frac{m_{1}}{g_{1}}, t_{2}\right)=1 \\
g_{1} \text { is even }}}\left(\frac{1}{m_{1} t_{1}}\right)^{1-2 s}\left(g_{1} t_{1}\right)^{1-2 s}\left(\frac{Y}{g_{1}^{2} t^{2}}\right)^{1-s} \frac{\phi\left(m_{1} g_{1}^{-1}\right)}{m_{1} g_{1}^{-1}} \frac{t_{2}^{-2 s}}{1-s}\right. \\
& \left.+\sum_{\substack{g_{1} \mid m_{1} \\
\left(\frac{m_{1}}{g_{1}}, t_{2}\right)=1 \\
g_{1} \text { is odd }}}\left(\frac{1}{m_{1} t_{1}}\right)^{1-2 s}\left(g_{1} t_{1}\right)^{1-2 s}\left(\frac{Y}{g_{1}^{2} t^{2}}\right)^{1-s} \frac{1}{2} \frac{\phi\left(m_{1} g_{1}^{-1}\right)}{m_{1} g_{1}^{-1}} \frac{t_{2}^{-2 s}}{1-s}\right\} \\
& +O\left(\sum_{t_{1} \mid t} \sum_{\substack{g_{1} \\
\left(g_{1}, t_{2}\right)=1}} \sum_{\substack{h_{1}=1 \\
\left(h_{1}, t_{2}\right)=1}}^{\infty} \frac{g_{1} t_{1}}{\left(g_{1} h_{1} t_{1}\right)^{1+2 \eta}} Y^{\eta} \mathrm{d}\left(h_{1}\right)(|s|+1)\right) \\
& =\sum_{t_{1} \mid t}\left\{\sum_{\substack{g_{1} \\
\left(g_{1}, t_{2}\right)=1 \\
g_{1} \text { is seven }}} \sum_{\substack{h_{1}=1 \\
\left(h_{1}, t_{2}\right)=1}}^{\infty} \frac{1}{h_{1}^{1-2 s}} \frac{Y^{1-s}}{1-s}\left(\frac{1}{g_{1} t}\right)^{2-2 s} \frac{\phi\left(h_{1}\right)}{h_{1}}\left(\frac{t_{1}}{t}\right)^{2 s}\right. \\
& \left.+\sum_{\substack{g_{1} \\
\left(g_{1}, 2_{2}\right)=1 \\
g_{1} \text { is odd }}} \sum_{\substack{h_{1}=1 \\
\left(h_{1}, t_{2}\right)=1}}^{\infty} \frac{1}{h_{1}^{1-2 s}} \frac{Y^{1-s}}{1-s}\left(\frac{1}{g_{1} t}\right)^{2-2 s} \frac{\phi\left(h_{1}\right)}{h_{1}}\left(\frac{t_{1}}{t}\right)^{2 s}\right\} \\
& +O\left(Y^{\eta}\left(\sum_{t_{1} \mid t}\right)(|s|+1)\right)
\end{aligned}
$$




$$
\begin{aligned}
& =\frac{1}{t^{2}} \sum_{t_{1} \mid t} t_{1}^{2 s}\left\{\sum_{\substack{g_{1} \\
\left(g_{1}, t_{2}\right)=1 \\
g_{1} \text { is seven }}}\left(\frac{1}{g_{1}}\right)^{2-2 s} \sum_{\substack{h_{1}=1 \\
\left(h_{1}, t_{2}\right)=1}}^{\infty} \frac{\phi\left(h_{1}\right)}{h_{1}^{2-2 s}} \frac{Y^{1-s}}{1-s}\right. \\
& \left.+\frac{1}{2} \sum_{\substack{g_{1} \\
\left(g_{1}, z_{2}\right)=1}}\left(\frac{1}{g_{1} \text { is odd }}\right)^{2-2 s} \sum_{\substack{h_{1}=1 \\
\left(h_{1}, t_{2}\right)=1}}^{\infty} \frac{\phi\left(h_{1}\right)}{h_{1}^{2-2 s}} \frac{Y^{1-s}}{1-s}\right\} \\
& +O\left(Y^{\eta}\left(\sum_{t_{1} \mid t} \frac{1}{t_{1}}\right)(|s|+1)\right) \\
& =\frac{1}{t^{2}} \sum_{t_{1} \mid t} t_{1}^{2 s}\left(\frac{1}{2}+\frac{1}{2}\left(\frac{1}{2}\right)^{2-2 s}\right) \zeta(1-2 s) \prod_{p \mid t_{2}}\left(1-p^{-1+2 s}\right) \frac{Y^{1-s}}{1-s} \\
& +O\left(Y^{1 / 2}\left(\sum_{t_{1} \mid t} \frac{1}{t_{1}}\right)^{(|s|+1)}\right)
\end{aligned}
$$

if we choose $\eta=\frac{1}{2}+\frac{1}{\log Y}$.

Therefore,

$$
\begin{aligned}
I_{\text {main }}= & -\frac{1}{2 \pi i} \frac{1}{2 t^{2}}\left\{\int_{(-\eta)} \sum_{t_{1} \mid t} t_{1}^{2 s} \prod_{p \mid t_{2}}\left(1-p^{-1+2 s}\right) \frac{Y^{1-s}}{1-s}\left(1+\left(\frac{1}{2}\right)^{2-2 s}\right) \zeta(1-2 s)\right. \\
& \left.+O(\pi X)^{s} \frac{\Gamma\left(\frac{1}{4}-\frac{s}{2}\right)}{\Gamma\left(\frac{1}{4}+\frac{s}{2}\right)} \Gamma(s) d s\right\} \\
= & -\frac{Y}{2 t^{2}} \frac{1}{2 \pi i}\left\{\int_{(-\eta)} \sum_{t_{1} \mid t} t_{1}^{2 s} \prod_{p \mid t_{2}}\left(1-p^{-1+2 s}\right) \frac{1}{1-s}\left(1+\left(\frac{1}{2}\right)^{2-2 s}\right) \zeta(1-2 s)\right. \\
& \left.+O\left(Y_{t_{1} \mid t} \frac{1}{t_{1}}\right)\right) \\
& \left.\times\left(\frac{\pi X}{Y}\right)^{s} \frac{\Gamma\left(\frac{1}{4}-\frac{s}{2}\right)}{\Gamma\left(\frac{1}{4}+\frac{s}{2}\right)} \Gamma(s) d s\right\}
\end{aligned}
$$

Recall that $t=t_{1} t_{2}$. Now use the Laurent expansions at $s=0$ :

$$
\begin{gathered}
\zeta(1-2 s)=-\frac{1}{2 s}+\gamma+\cdots, \\
\Gamma(s)=\frac{1}{s}-\gamma+\cdots,
\end{gathered}
$$




$$
\begin{aligned}
\frac{1}{1-s}\left(\frac{\pi X}{Y}\right)^{s} \frac{\Gamma\left(\frac{1}{4}-\frac{s}{2}\right)}{\Gamma\left(\frac{1}{4}+\frac{s}{2}\right)} & =1+\left(1-\frac{\Gamma^{\prime}}{\Gamma}\left(\frac{1}{4}\right)+\log \pi+\log \left(\frac{X}{Y}\right)\right) s+\cdots, \\
1+\left(\frac{1}{2}\right)^{2-2 s} & =\frac{5}{4}+\frac{\log 4}{4} s+\cdots, \\
\sum_{t_{1} \mid t} t_{1}^{2 s} \prod_{p \mid t_{2}}\left(1-p^{-1+2 s}\right) & =\sum_{t_{1} \mid t} \frac{\phi\left(t_{2}\right)}{t_{2}}+\sum_{t_{1} \mid t} \frac{\phi\left(t_{2}\right)}{t_{2}}\left(2 \log t_{1}-\sum_{p \mid t_{2}} \frac{2 \log p}{p-1}\right) s+\cdots
\end{aligned}
$$

Moving the line of integration from $\Re(s)=-\eta$ to the right to the line

$$
\Re(s)=\frac{1}{2}-\frac{1}{\log (Y / X)}
$$

and picking up the residue at $s=0$, we have

$$
\begin{array}{r}
I_{\text {mair }}=\frac{Y}{t^{2}}\left(\sum_{t_{1} \mid t} \frac{\phi\left(t_{1}\right)}{t_{1}}\right)\left(-\frac{5}{16} \log \left(\frac{X}{Y}\right)-\frac{5}{16} \log \pi+\frac{15}{16} \gamma-\frac{5}{16}+\frac{5}{16} \frac{\Gamma^{\prime}}{\Gamma}\left(\frac{1}{4}\right)\right. \\
\left.-\frac{\log 4}{16}-\frac{5}{8}\left(\log t_{2}\right)+\frac{5}{8} \sum_{p \mid t_{1}} \frac{\log p}{p-1}\right)+O\left(Y^{1 / 2} X^{1 / 2} \frac{1}{t^{2}} \sum_{t_{1} \mid t} t_{1}\right) .
\end{array}
$$

Now for the error term $I_{\text {error }}$, which corresponds to nonsquares $n$, we have

$$
\begin{aligned}
I_{\text {error }} & =-\frac{1}{2 \pi i} \int_{(-\eta)} \sum_{\substack{n=1 \\
n \text { noosquare }}}^{\infty} \frac{1}{n^{1 / 2-s}} \sum_{\substack{0<D \leq Y \\
D \equiv 0,1(\bmod 4) \\
i^{2} \mid D}} \varepsilon_{D}(n) D^{-s} X^{s} \pi^{s} \frac{\Gamma\left(\frac{1}{4}-\frac{s}{2}\right)}{\Gamma\left(\frac{1}{4}+\frac{s}{2}\right)} \Gamma(s) d s \\
& =-\frac{1}{2 \pi i} \int_{(-\eta)} \sum_{\substack{n \leq U \\
n \text { nossquare }}}+\frac{1}{2 \pi i} \int_{(-\eta)} \sum_{\substack{n>U \\
n \text { nossquare }}}=I_{\text {error } 1}+I_{\text {error } 2}
\end{aligned}
$$

where $U=Y^{4} / X^{2}$.

For the first integral $I_{\text {error } 1}$, using Polya-Vinogradov inequality and partial summation, we have

$$
\left|\sum_{\substack{0<D \leq \gamma \\ D \equiv 0,1(\bmod 4) \\ t^{2} \mid D}} \varepsilon_{D}(n) D^{-s}\right|=O\left(\sqrt{n}(\log n) \gamma(n) Y^{\eta}\right)
$$

Then

$$
I_{\text {error 1 }}=O\left(Y^{\eta} X^{-\eta} \sum_{\substack{n \leq U \\ n \text { noseguere }}} \frac{(\log n) \gamma(n)}{n^{\eta}}\right)
$$

by the exponential decay of the Gamma function. 
But

$$
\begin{aligned}
\sum_{\substack{n \leq U \\
n \text { nonsquare }}} \frac{(\log n) \gamma(n)}{n^{\eta}} & =\sum_{\substack{n \leq U \\
n \text { nongquare }}} \frac{\log n}{n^{\eta}} \sum_{g^{2} \mid n} 1 \\
& =\sum_{\substack{g \leq \sqrt{U} \\
n \text { Donsquare }}} \frac{1}{g^{2 \eta}} \sum_{n \leq U / g^{2}} \frac{\log n}{n^{\eta}}=O\left((\log U) U^{1-\eta}\right),
\end{aligned}
$$

then

$$
I_{\text {error } 1}=\left(\left(\frac{Y}{X}\right)^{\eta} U^{1-\eta}(\log U)\right)
$$

If we choose $\eta=1-\frac{1}{\log \left(Y^{3} / X\right)}$, then

$$
I_{\text {error } 1}=O\left(\left(\frac{Y}{X}\right)^{\eta} \frac{Y^{4-4 \eta}}{X^{2-2 \eta}}(\log Y)\right)=O\left(\frac{Y}{X}(\log Y)\right) .
$$

Next, for the integral $I_{\text {error } 2 \text {, using the trivial bound, we have }}$

$$
\begin{aligned}
I_{\text {error } 2} & =O\left(\sum_{\substack{n>U \\
n \text { noosquare }}} \frac{1}{n^{1 / 2+\eta}} Y^{1+\eta} X^{-\eta}\right) \\
& =O\left(Y^{1+\eta} X^{-\eta} U^{1 / 2-\eta}\right)=O\left(\frac{Y^{3}}{X}\left(\frac{X}{Y^{3}}\right)^{\eta}\right)=O(1)
\end{aligned}
$$

Therefore

$$
I_{\text {error }}=O\left(\frac{Y}{X}(\log Y)\right)
$$

In summary, we have

$$
\begin{aligned}
\sum_{\substack{0<D \leq Y \\
D \equiv 0,1(\bmod 4) \\
t^{2} \mid D}} L\left(\frac{1}{2}, \varepsilon_{D}\right)= & \frac{Y}{t^{2}} \sum_{t_{1} \mid t} \frac{\phi\left(t_{1}\right)}{t_{1}}\left\{\frac{5}{16} \log Y+\frac{5}{4} \gamma-\frac{5}{16} \log \pi-\frac{\log 4}{8}-\frac{5}{16}\right. \\
& \left.+\frac{5}{16} \frac{\Gamma^{\prime}}{\Gamma}\left(\frac{1}{4}\right)-\frac{5}{4} \log \left(\frac{t}{t_{1}}\right)+\frac{5}{4} \sum_{p \mid t_{1}} \frac{\log p}{p-1}\right\} \\
& +O\left(Y^{1 / 2} X^{1 / 2} \frac{1}{t^{2}}\left(\sum_{t_{1} \mid t} t_{1}\right)\right)+O\left(\frac{Y}{X}(\log Y)\right) \\
& +O\left(\frac{Y}{X} \sum_{t_{1} \mid t} \frac{1}{t_{1}}\right)+O\left(\mathrm{~d}(t) X^{1 / 2}(\log X)^{2}\right)+O(X \log X) .
\end{aligned}
$$

The error term is $O\left(Y^{2 / 3}(\log Y)\right)$ if we choose $X=Y^{1 / 3}$. 
In the case that $(t, 2) \neq 1$, we have

$$
\begin{array}{r}
\sum_{\substack{0<D \leq Y \\
D \equiv 0,1(\bmod 4) \\
t^{2} \mid D}} L\left(\frac{1}{2}, \varepsilon_{D}\right)=\frac{Y}{t^{2}} \sum_{t_{1} \mid t} \frac{\phi\left(t_{1}\right)}{t_{1}}\left\{\frac{1}{2} \log Y+2 \gamma-\frac{\log \pi}{2}-\frac{1}{2}+\frac{1}{2} \frac{\Gamma^{\prime}}{\Gamma}\left(\frac{1}{4}\right)\right. \\
\left.-2 \log \left(\frac{t}{t_{1}}\right)+2 \sum_{p \mid t_{1}} \frac{\log p}{p-1}\right\}+O\left(Y^{2 / 3}(\log Y)\right) .
\end{array}
$$

This concludes the proof of the theorem.

Remark. For small values of $t$, we used the Polya-Vinogradov inequality to get upper bounds for character sums. For large values of $t$, other inequalities can be used, and we can get the error term in Theorem 1 divided by a function of $t$, but the exponent of $Y$ can be larger.

Taking $t=1$, we have

\section{Corollary.}

$$
\begin{aligned}
\sum_{\substack{0<D \leq Y \\
D \equiv 0,1(4)}} L\left(\frac{1}{2}, \varepsilon_{D}\right)= & \frac{5}{16} Y \log Y+\frac{5}{16} Y\left(4 \gamma-\log \pi-\frac{2 \log 4}{5}-1+\frac{\Gamma^{\prime}}{\Gamma}\left(\frac{1}{4}\right)\right) \\
& +O\left(Y^{2 / 3} \log Y\right) .
\end{aligned}
$$

For the case $D \in[-Y, 0)$, the corresponding functional equation is

$$
L\left(\frac{1}{2}+s, \varepsilon_{D}\right)=|D|^{s} \pi^{s} \frac{\Gamma\left(\frac{3}{4}-\frac{s}{2}\right)}{\Gamma\left(\frac{3}{4}+\frac{s}{2}\right)} L\left(\frac{1}{2}-s, \varepsilon_{D}\right)
$$

then we have

Theorem 2.

$$
\begin{aligned}
& \sum_{\substack{-Y<D<0 \\
D=0,1(\bmod ) \\
t^{2} \mid D}} L\left(\frac{1}{2}, \varepsilon_{D}\right) \\
& =\left\{\begin{array}{r}
\frac{5}{16} \frac{Y}{t^{2}} \sum_{t_{1} \mid t} \frac{\phi\left(t_{1}\right)}{t_{1}}\left(\log Y+4 \gamma-\log \pi-\frac{2 \log 4}{5}-1+\frac{\Gamma^{\prime}}{\Gamma}\left(\frac{3}{4}\right)\right. \\
\left.-4 \log \left(\frac{t}{t_{1}}\right)+4 \sum_{p \mid t_{1}} \frac{\log p}{p-1}\right)+O\left(Y^{2 / 3}(\log Y)\right) \\
\frac{1}{2} \frac{Y}{t^{2}} \sum_{t_{1} \mid t} \frac{\phi\left(t_{1}\right)}{t_{1}}\left(\log Y+4 \gamma-\log \pi-1+\frac{\Gamma^{\prime}}{\Gamma}\left(\frac{3}{4}\right) \quad \text { if } t \text { is odd },\right. \\
\left.-4 \log \left(\frac{t}{t_{1}}\right)+4 \sum_{p \mid t_{1}} \frac{\log p}{p-1}\right)+O\left(Y^{2 / 3}(\log Y)\right)
\end{array}\right.
\end{aligned}
$$




\section{ACKNOWLEDGMENTS}

The author would like to thank Professor Ram Murty for his suggestions and discussions during this work. He also thanks the referee for various helpful comments.

\section{REFERENCES}

1. H. Davenport, Multiplicative number theory, second ed., Springer-Verlag, New York, 1980.

2. D. Goldfeld and J. Hoffstein, Eisenstein series of $\frac{1}{2}$-integral weight and the mean value of real Dirichlet $L$-series, Invent. Math. 80 (1985), 185-205.

3. D. Goldfeld and C. Viola, Mean values of L-functions associated to Elliptic, Fermat and other curves at the center of the critical strip, J. Number Theory 11 (1979), 305-320.

4. M. Jutila, On the mean value of $L\left(\frac{1}{2}, \chi\right)$ for real characters, Analysis $1(1981), 149-161$.

5. W. Kohnen and D. Zagier, Values of $L$-series of modular forms at the center of the critical strip, Invent. Math. 64 (1981), 175-198.

6. M. R. Murty and V. K. Murty, $A$ variant of the Bombieri-Vinogradov Theorem, CMS Conf. Proc., vol. 7, Amer. Math. Soc., Providence, RI, 1987, pp. 243-272.

7. 447-475.

8. C. L. Siegel, The average measure of quadratic forms with given determinant and signature, Ann. of Math. (2) 45 (1944), 667-685.

9. A. I. Vinogradov and L. A. Tahtadzjan, On analogues of the Vinogradov-Gauss formula, Soviet Math. Dokl. 22 (1980), 555-559.

10. D. Zagier, Modular forms whose Fourier coefficients involve Zeta functions of quadratic fields, Modular Forms of One Variable VI (J. P. Serre and D. Zagier, eds.), Lecture Notes in Math., vol. 627, Springer-Verlag, New York, 1977, pp. 105-169.

The Fields Institute for Research in Mathematical Sciences, 185 Columbia Street West, Waterloo, Ontario, Canada N2L 5Z5

E-mail address: maiefields.uwaterloo.ca 